\title{
Two loops calculation in chiral perturbation theory and the unitarization program of current algebra
}

\author{
J. Sá Borges*, J. Soares Barbosa ${ }^{\dagger}$ and M. D. Tonasse ${ }^{\ddagger}$ \\ Instituto de Física, Universidade do Estado do Rio de Janeiro, Rua São Francisco Xavier 524, 20550-013 - Rio de Janeiro, RJ \\ Brazil
}

(October 9, 2018)

\begin{abstract}
In this paper we compare two loop Chiral Perturbation Theory (ChPT) calculation of pion-pion scattering with the unitarity second order correction to the current algebra soft-pion theorem. It is shown that both methods lead to the same analytic structure for the scattering amplitude. Current algebra; Unitarity.
\end{abstract}

PACS numbers: 12.39.Fe, 11.30.Rd, 13.75.Lb

\section{INTRODUCTION}

In the early sixties, many results for low energy meson physics have been derived from the assumptions of a local chiral $\mathrm{SU}(2) \times \mathrm{SU}(2)$ algebra of vector and axial vector current densities together with the partial conservation hypothesis (PCAC) relating the derivative of the axial vector current to the pion field. By itself, the PCAC relation can not help one to extend the applicability of current algebra method to the energy corresponding to meson-meson resonances. Nevertheless, a method was invented to obtain results for process were mesons are not "soft particles". We are referring to the hard meson methods of current algebra [1]. Even ignoring the underlying theory, the chiral current algebra implies a set of Ward identities and the method consists in solving the system of Ward identities under the general principles of analyticity, crossing and elastic unitarity.

In 1979, Weinberg suggested that it is possible to summarize these previous results in a phenomenological Lagrangian which incorporates all the constraints coming from chiral symmetry of the underlying theory [2]. In a set of very important and fundamental papers, Gasser and Leutwyller have developed Chiral Perturbation Theory (ChPT) which allows one to compute many different Green functions involving low energy pions [3]. One of the main obstacles for the applications of ChPT to high energies lies in the issue of unitarity. Several methods have been proposed to extend the range of energies where ChPT could be applied. These methods include the Padé expansion [4], the inverse amplitude method [5] and the introduction of fields describing resonances [6].

Let us remember, however that, in order to treat pion-pion scattering amplitude obtained by the hard meson method based on the Ward identity technique, one of us have introduced the constraints of elastic unitarity for partial waves [7]. We will call this approach by unitarization program of current algebra (UPCA). It was applied to obtain first order corrections (QU1) to the soft-pion $\pi \pi$ Weinberg amplitude [8] as well as to calculate second order corrections (QU2) to the referred amplitude [9].

The ChPT calculation of pion-pion scattering to one loop was performed in Ref. [3] and, only recently, two loops calculation appeared in the literature [10]. Our aim is to compare UPCA and ChPT calculations. In a previous paper, we have shown that one loop ChPT is equivalent to QU1 [9] and in the present paper, we will compare QU2 amplitude with ChPT Lagrangian two loops calculation performed in Ref. [10]. We will conclude that, as conjectured by one of us [9], the two approaches are equivalent. In the next section we will recall the comparison at one loop level and in the section three this comparison is extended to the two loops case. In the section four we present the conclusions.

\footnotetext{
*E-mail: Borges@vax.fis.uerj.br

${ }^{\dagger}$ E-mail: Soares@vax.fis.uerj.br

${ }^{\ddagger}$ E-mail: Tonasse@vax.fis.uerj.br
} 


\section{FIRST ORDER CORRECTED BY UPCA AMPLITUDE AND ONE LOOP CHPT}

Let us remember the main points of the UPCA. The starting point in our derivation was an exact hard-pion expression for the correlation function of four currents, with the quantum numbers of the pion, in terms of three- and two-point functions. From this expression, by using vertex and propagator estimates, we could reobtain the so-called soft-pion Weinberg amplitude, namely:

$$
A^{C A}(s, t, u)=\frac{1}{F_{\pi}^{2}}\left(s-m^{2}\right) .
$$

The remaining of the amplitude reflects the difference between soft- and hard-meson result. In the UPCA one estimates the behavior of form factors and propagators at low energies and assumes that, for instance, for small values of its argument, the scalar pion form factor, $F_{I}$, is of the same order of magnitude as current algebra amplitudes near threshold. This can be obtained by setting, for $x \simeq m^{2}, \quad F_{I}(x) \simeq 1+f_{I}^{(1)}(x)+O\left(\epsilon^{2}\right)$, for $I=0$ and 2 , where the superscript "(1)" denotes the order $\epsilon=m^{2} / M^{2}, M$ being of order of magnitude of vector meson masses.

To construct unitarized amplitude, we have observed that current algebra gives real partial waves. The unitarization method must provide an imaginary part to the corrected partial wave. Thus, at the first order of the calculation, by the optical theorem, one must have

$$
\operatorname{Im} t_{\ell I}^{(1)}(s)=\frac{1}{32 \pi} \sigma(s) t_{\ell I}^{C A}(s)^{2},
$$

where $t_{\ell I}^{C A}$ is the soft-pion $\ell$ partial wave, isospin $I$, Weinberg amplitude obtained from Eq. (1) and

$$
\sigma(s)=\sqrt{\frac{s-4 m^{2}}{s}} .
$$

In the program, we work with exact total amplitude expression which follows from the Ward identities, and we use the implications of elastic unitarity for form factors and propagators in a peculiar way. For instance, from the relations valid for the scalar form factor and scalar propagator, namely:

$$
\operatorname{Im} F_{I}(s)=\frac{1}{32 \pi} \sigma(s) T_{I}^{*}(s) F_{I}(s) \quad \text { and } \operatorname{Im} \Delta_{I}(s)=\frac{1}{32 \pi} \sigma(s)\left|F_{I}(s)\right|^{2}, I=0 \text { and } 2
$$

we obtain within the first order of the approximation:

$$
\operatorname{Im} f_{0}^{(1)}(s)=\frac{1}{32 \pi} \sigma(s) t_{00}^{C A}(s) \quad \text { and } \operatorname{Im} \quad \delta_{I}^{(1)}(s)=\frac{1}{32 \pi} \sigma(s),
$$

where $t_{00}^{C A}$ is the current algebra isospin zero S-wave $\pi \pi$ amplitude,

$$
t_{00}^{C A}(s)=\frac{1}{F_{\pi}^{2}}\left(2 s-m^{2}\right)
$$

Considering the known imaginary part of each function entering into the amplitude, the method consists in obtaining their real parts by the dispersion relation technique. To converge, dispersion integrals need subtraction which are model free parameters. They can be fixed by fitting experimental data.

In this way, the first order corrected amplitude, QU1, derived in the context of UPCA [7], can be written in the following form:

$$
\begin{aligned}
F_{\pi}^{4} A_{Q U 1}^{(1)}(s, t, u)= & \frac{1}{3}\left(2 s-m^{2}\right) \Phi_{0}^{(1)}(s)-\frac{1}{3}\left(2 m^{2}-s\right) \Phi_{2}^{(1)}(s)+\frac{1}{2} \xi_{1}\left(s-2 M^{2}\right)^{2}+ \\
& {\left[\frac{1}{2}\left(2 m^{2}-t\right) \Phi_{2}^{(1)}(t)+(s-u) \Phi_{1}^{(1)}(t)-\frac{1}{4} \xi_{2}\left(t-2 M^{2}\right)^{2}+(t \leftrightarrow u)\right] }
\end{aligned}
$$

with:

$$
\begin{aligned}
& \Phi_{0}^{(1)}(x)=\left(2 x-m^{2}\right)\left(g(x)+\alpha_{0}\right), \quad \Phi_{2}^{(1)}(x)=\left(2 m^{2}-x\right)\left(g(x)+\alpha_{2}\right), \\
& \Phi_{1}^{(1)}(x)=\frac{1}{3}\left(x-4 m^{2}\right) g(x)-\frac{1}{3}\left(2 x \alpha_{1}-4 m^{2} g(0)\right)
\end{aligned}
$$


where:

$$
32 \pi^{2} g(s)=\left(s-4 m^{2}\right) \int_{4 m^{2}}^{\infty} d x \frac{\sigma(x)}{\left(x-4 m^{2}\right)(x-s)}=\sigma(s) \ln \frac{\sigma(s)-1}{\sigma(s)+1} .
$$

On the other hand, the one loop elastic pion scattering obtained from ChPT Lagrangian [3] is:

$$
\begin{aligned}
F_{\pi}^{4} A_{C h P T}^{(1)}(s, t, u)= & \frac{1}{2}\left(s^{2}-m^{4}\right) \bar{J}(s)+ \\
& \frac{1}{6}\left[\left(t(t-u)-2 m^{2} t+4 m^{2} u-2 m^{4}\right) \bar{J}(t)+(t \leftrightarrow u)\right]+ \\
& {\left[2\left(\bar{\ell}_{1}-4 / 3\right)\left(s-2 m^{2}\right)^{2}+\left(\bar{\ell}_{2}-5 / 6\right)\left(s^{2}+(t-u)^{2}\right)+\right.} \\
& \left.12 m^{2} s\left(\bar{\ell}_{4}-1\right)-3\left(\bar{\ell}_{3}+4 \bar{\ell}_{4}-5\right) m^{4}\right] / 96 \pi^{2} .
\end{aligned}
$$

We can identify the function $\bar{J}(x)$ with $2[g(x)-g(0)]$ and we have verified that the polynomial coefficients of these functions are the same. We have then concluded that the above amplitude has the same analytical structure than QU1. Each approach have its free parameters: the model free parameters of QU1 are $\xi^{\prime} s$ and $\alpha^{\prime} s$ linear combinations and the free parameters of ChPT are $\bar{\ell}^{\prime} s$ linear combinations. In UPCA the free parameters are subtraction constants, inherent to dispersion relation technique, and in ChPT they come from tadpole graphs and tree graphs of order $\mathrm{O}\left(p^{4}\right)$.

From this comparison we have shown, in a recent letter, that one loop ChPT amplitude can fit experimental S- and P- waves up to the resonance region by adjusting only two parameters, namely $\bar{\ell}_{1}$ and $\bar{\ell}_{2}$ [1].

In the next section, we will compare the second order corrected by UPCA amplitude, QU2, with two loops calculation [10]. To do this, we will need one loop partial wave corresponding to the ChPT amplitude given above. For this, one expands the combinations with definite isospin in the s-channel into partial waves:

$$
T_{I}(s, t)=32 \pi \sum_{\ell=0}^{\infty}(2 \ell+1) P_{\ell}(\cos \theta) t_{\ell I}^{(1)}(s), \quad I=0,1 \text { and } 2 .
$$

Using Eq. (㺼), the resulting one loop P-wave amplitude is:

$$
\begin{aligned}
t_{11}^{(1)}(s)= & \frac{1}{18 F_{\pi}^{4}}\left(s-4 m^{2}\right)^{2} \bar{J}(s)+\left\{\frac{m^{4}}{8}\left(\frac{s^{2}}{2}-\frac{13}{16} s m^{2}-m^{4}\right) \frac{L^{2}(s)}{\left(s-4 m^{2}\right)^{2}}\right. \\
& +\left(\frac{s^{3}}{288}-\frac{1}{18} s^{2} m^{2}+\frac{1}{4} s m^{4}-\frac{m^{6}}{8}\right) \frac{L(s)}{\sigma\left(s-4 m^{2}\right)} \\
& -\frac{1}{864\left(s-4 m^{2}\right)}\left(s^{3}+37 s^{2} m^{2}-149 s m^{4}+120 m^{6}\right) \\
& \left.+\frac{s-4 m^{2}}{288}\left[\left(2 \bar{\ell}_{2}-2 \bar{\ell}_{1}-1\right) s+8 m^{2}\right]\right\} \frac{1}{F_{\pi}^{4} \pi^{2}},
\end{aligned}
$$

the isospin $I=0$ one loop S-wave is:

$$
\begin{aligned}
t_{00}^{(1)}(s)= & \frac{1}{2 F_{\pi}^{4}}\left(2 s-m^{2}\right)^{2} \bar{J}(s)+\frac{1}{F_{\pi}^{4} \pi^{2}}\left\{\frac{m^{4}}{8}\left(s-\frac{25}{6} m^{2}\right) \frac{L^{2}(s)}{\left(s-4 m^{2}\right)}\right. \\
& -\left(\frac{7}{144} s^{2}-\frac{5}{18} s m^{2}+\frac{25}{48} m^{4}\right) \frac{L(s)}{\sigma}+\left(\frac{11}{144} \bar{\ell}_{1}+\frac{7}{72} \bar{\ell}_{2}-\frac{7}{96}\right) s^{2} \\
& \left.-\left(\frac{5}{18}\left(\bar{\ell}_{1}+\bar{\ell}_{2}\right)-\frac{1}{4} \bar{\ell}_{4}-\frac{481}{432}\right) s m^{2}+\frac{m^{4}}{18}\left(\frac{11}{2} \bar{\ell}_{1}+7 \bar{\ell}_{2}-\frac{45}{16} \bar{\ell}_{3}-\frac{9}{4} \bar{\ell}_{4}+\frac{355}{16}\right)\right\}
\end{aligned}
$$

and the isospin $I=2$ one loop S-wave is:

$$
\begin{aligned}
t_{02}^{(1)}= & \frac{1}{2 F_{\pi}^{4}}\left(2 m^{2}-s\right)^{2} \bar{J}(s)+\frac{1}{F_{\pi}^{4} \pi^{2}}\left\{-\frac{m^{4}}{16}\left(s+\frac{m^{2}}{3}\right) \frac{L^{2}(s)}{\left(s-4 m^{2}\right)}\right. \\
& -\left(\frac{11}{288} s^{2}-\frac{1}{9} s m^{2}+\frac{m^{4}}{48}\right) \frac{L(s)}{\sigma} \\
& -\left(\frac{1}{72} \bar{\ell}_{1}+\frac{1}{18} \bar{\ell}_{2}+\frac{5}{192}\right) s^{2}-\left(\frac{1}{36} \bar{\ell}_{1}+\frac{7}{36} \bar{\ell}_{2}+\frac{1}{8} \bar{\ell}_{4}-\frac{527}{864}\right) s m^{2} \\
& \left.+\left(\frac{1}{18} \bar{\ell}_{1}+\frac{2}{9} \bar{\ell}_{2}-\frac{1}{16} \bar{\ell}_{3}+\frac{1}{4} \bar{\ell}_{4}-\frac{1}{9}\right) m^{4}\right\} .
\end{aligned}
$$

In these expressions we have included the contributions from $\bar{J}\left(4 m^{2}\right)$ which is lacking in the expressions of partial waves given in the Sec. 2 of the Ref. [11] and we have corrected an overall sign. 


\section{SECOND ORDER CORRECTED BY UPCA AMPLITUDE AND TWO LOOPS CHPT}

We have shown that the first order correction to the soft pion amplitude (QU1) is equivalent to one loop ChPT scattering amplitude and, in addition, we have given the tools for constructing the next order unitarity corrections (QU2) [9]. The formula (3.10) of Ref. [9] can be written as:

$$
\begin{aligned}
F_{\pi}^{6} A_{Q U 2}^{(2)}(s, t, u)= & \frac{1}{3}\left(2 s-m^{2}\right) \Phi_{0}^{(2)}(s), \\
& -\frac{1}{3}\left(2 m^{2}-s\right) \Phi_{2}^{(2)}(s)\left[\frac{1}{2}\left(2 m^{2}-t\right) \Phi_{2}^{(2)}(t)+(s-u) \Phi_{1}^{(2)}(t)+(t \leftrightarrow u)\right] .
\end{aligned}
$$

We can relate the above expression with the formula (3) obtained as a consequence of the Goldstone nature of the pion [12], namely:

$$
F_{\pi}^{4} W_{I}(s)=\frac{1}{32 \pi} t_{I}^{C A}(s) \Phi_{I}^{(2)}(s), \quad \text { for } I=0,2 \quad \text { and } \quad F_{\pi}^{4} W_{1}(s)=\frac{1}{48 \pi} \Phi_{1}^{(2)}(s) .
$$

The functions $W_{I}(s)$ are analytic except for a cut singularity at $s \geq 4 m^{2}$. Their discontinuities are directly related with the discontinuities of the functions $\Phi_{I}(s)$.

We would like to emphasize that, the general structure of the UPCA solution comes from the Ward identity method and the hard meson technique implies that the amplitude is written in terms of form factors and propagators. We stress that the UPCA is based on the implications of elastic unitarity relations for form factors and propagators, and not for partial waves themselves.

The consequences, of using Eq. (2), for instance for scalar form factors and propagators, to second order of the approximation, are:

$$
\operatorname{Im} f_{I}^{(2)}(s)=\frac{1}{32 \pi} \sigma(s)\left(\operatorname{Re} t_{I}^{(1)}(s)+t^{C A}(s) \operatorname{Re} f_{I}^{(1)}(s)\right), \quad \operatorname{Im} \delta_{I}^{(2)}(s)=\frac{1}{32 \pi} 2 \operatorname{Re} f_{I}^{(1)}(s)
$$

with $I=0$ and 2 . The vector form factor and vector propagator are obtained in a similar way. The functions $\Phi_{I}^{(2)}(s)$, constructed from form factors and propagators are then discontinued on the right hand cut as follows:

$$
\operatorname{Im} \Phi_{I}^{(2)}(x)=\frac{1}{32 \pi} \sigma(x) 2 \operatorname{Re} t_{I}^{(1)}(x), \quad \text { for } I=0,1 \text { and } 2
$$

and $\operatorname{Re} t^{(1)}$ stands for the real parts of the functions in Eq. (5). Using dispersion relation technique we obtain:

$$
\Phi_{I}^{(2)}(s)=p_{I}(s) Z(s)+q_{I}(s) G(s)+r_{I}(s) g(s)+P_{I}(s) \text { for } I=0,1 \text { and } 2 .
$$

The polynomials $p_{I}(s), q_{I}(s), r_{I}(s)$, and $P_{I}(s)$, for each value of total isospin $I$, are given in the Appendix. The function $g(s)$ is given in (3)and

$$
\begin{aligned}
32 \pi^{2} G(s) & =\left(s-4 m^{2}\right) \int_{4 m^{2}}^{\infty} d x \frac{\sigma(x) \operatorname{Re} g(x)}{\left(x-4 m^{2}\right)(x-s)}=\frac{1}{64 \pi^{2}} \sigma^{2}(s) L^{2}(s)+\frac{\pi^{2}}{3} \sigma^{2}(s), \\
\left(32 \pi^{2}\right)^{2} Z(s) & =\left(s-4 m^{2}\right) \int_{4 m^{2}}^{\infty} d x \frac{\sigma(x)}{\left(x-4 m^{2}\right)(x-s)}(L(x)+i \pi)^{2}=\frac{1}{3} L(s)\left(L^{2}(s)+\pi^{2}\right)
\end{aligned}
$$

where

$$
L(s)=\ln \frac{\sigma(s)-1}{\sigma(s)+1}
$$

The amplitude $A_{Q U 2}^{(2)}$ is then written in terms of powers of $L(s)$ and $L(t)$ and contains the free parameters $\bar{\ell}_{1}$, $\bar{\ell}_{2}$ and the values of the subtraction constants $\left(\Phi_{I}\right.$ and its derivatives at $\left.s=4 m^{2}\right)$.

On the other hand, ChPT amplitude calculated at two loops level [6] with $m_{\pi}=1$ is:

$$
F_{\pi}^{4} A_{C h P T}^{(2)}(s, t, u)=F^{(2)}(s)+G^{(2)}(s, t)+G^{(2)}(s, u),
$$


with:

$$
\begin{aligned}
F^{(2)}(s) & =\bar{J}(s)\left\{\frac{1}{16 \pi^{2}}\left(\frac{503}{108} s^{3}-\frac{929}{54} s^{2}+\frac{887}{27} s-\frac{140}{9}\right)+b_{1}(4 s-3)+b_{2}\left(s^{2}+4 s-4\right)\right. \\
& \left.+\frac{1}{3} b_{3}\left(8 s^{3}-21 s^{2}+48 s-32\right)+\frac{1}{3} b_{4}\left(16 s^{3}-71 s^{2}+112 s-48\right)\right\} \\
& +\frac{1}{18} K_{1}(s)\left[20 s^{3}-19 s^{2}+210 s-135-\frac{9}{16} \pi^{2}(s-4)\right] \\
& +\frac{1}{32} K_{2}(s)\left(s \pi^{2}-24\right)+\frac{1}{9} K_{3}(s)\left(3 s^{2}-17 s+9\right), \\
G^{(2)}(s, t) & =\bar{J}(t)\left\{\frac{1}{16 \pi^{2}}\left[\frac{412}{27}-\frac{s}{54}\left(t^{2}+5 t+159\right)-t\left(\frac{267}{216} t^{2}-\frac{727}{108} t+\frac{1571}{108}\right)\right]\right. \\
& +b_{1}(2-t)+\frac{1}{3} b_{2}(t-4)\left(t^{2}+s-5\right)-\frac{1}{6} b_{3}(t-4)^{2}(3 t+2 s-8) \\
& \left.+\frac{1}{6} b_{4}\left(2 s(3 t-4)(t-4)-32 t+40 t^{2}-11 t^{3}\right)\right\} \\
& +\frac{1}{36} K_{1}(t)\left[174+8 s-10 t^{3}+72 t^{2}-185 t-\frac{1}{16} \pi^{2}(t-4)(3 s-8)\right] \\
& +\frac{1}{9} K_{2}(t)\left[1+4 s+\frac{1}{64} \pi^{2} t(3 s-8)\right] \\
& +\frac{1}{9} K_{3}(t)\left(1+3 s t-s+3 t^{2}-9 t\right)+\frac{5}{3} K_{4}(t)(4-s-t) .
\end{aligned}
$$

In this expression $\bar{J}(s)=2(g(s)-g(0))$, and:

$$
\begin{aligned}
& K_{1}=\frac{L^{2}(s)}{\left(16 \pi^{2}\right)^{2}},\left(16 \pi^{2}\right)^{2} K_{2}=\sigma^{2} L^{2}(s)-4,\left(16 \pi^{2}\right)^{2} K_{3}=\frac{1}{s \sigma} L^{3}(s)+\frac{\pi^{2}}{s \sigma} L(s)-\frac{\pi^{2}}{2}, \\
& K_{4}=\frac{1}{s \sigma^{2}}\left(\frac{1}{2} K_{1}+\frac{1}{3} K_{3}+\frac{1}{16 \pi^{2}} \bar{J}+s \frac{\pi^{2}-6}{192 \pi^{2}}\right) .
\end{aligned}
$$

Our strategy to compare $A_{Q U 2}^{(2)}$ with $A_{C h P T}^{(2)}$ was to expand them in terms of $L(s)$ and $L(t)$ and then to confront its coefficients. We have checked that they are the same. With respect to the polynomials we also realize that the structure are the same, but clearly the coefficients are differents because they have different origins. These polynomials include the model free parameters to be used in order to fit the available experimental data [14].

\section{CONCLUSIONS}

Our aim is to compare Chiral Perturbation Theory (ChPT) calculations with the Unitarization Program of Current Algebra (UPCA). In previous works we have compared one loop ChPT with the first order corrected by UPCA results for pion-pion [9] and for kaon-pion scattering [13] and we have concluded that they lead to the same analytical structure for the amplitudes.

The two loops calculation of pion-pion scattering only recently appeared in the literature [10]. However, the tools for constructing second order corrections for the soft-pion current algebra result has been presented more than ten years ago [9]. In the present paper we compare these results and we show that, as it was conjectured [9], they have the same analytical structure. We will shortly present the tools for constructing next order UPCA correction to kaon-pion. ChPT to two loops for $K \pi$ are not yet available, but we expected that it will be equivalent to the UPCA result too.

In fact, the equivalence between the two approaches were expected. In the hard-meson method one starts from the chiral symmetric Ward identity exact result for the correlation function of four currents carrying pion quantum numbers. On the other hand, ChPT describes the low energy dynamics of fields realizing non-linearly chiral symmetry. Our unitarization program is based in the principles of analyticity, crossing and elastic unitarity which in turn are inherent to a field theory such as ChPT.

In the framework of the Generalized Chiral Perturbation Theory, it was shown how to implement elastic unitarity strating from the one loop partial waves. That procedure leads to equivalent $\mathrm{O}\left(p^{6}\right) \mathrm{ChPT}$ amplitude [12]. However, the main difference from our unitarization procedure is that it uses the consequences of elastic unitarity for the form factors and propagators rather than for the amplitudes themselves. 
Despite the fact that ChPT is a well stablished low energy effective theory for meson processes, we have shown here that the UPCA is a suitable alternative. One obstacle related to these two approaches is how to fix the free parameters which, in principle, are related to the parameters of the fundamental theory. At present, since this relationship is still lacking, the two approaches are both in same footing. In ChPT context we have shown that the one loop parameters $\bar{\ell}_{1}$ and $\bar{\ell}_{2}$ can be fixed by fitting S- and P-wave $\pi \pi$ phase-shifts [1]. The final expression of pion-pion ChPT amplitude up to two loops diagrams has six parameters. However, the D-wave amplitude from $A^{(2)}$ pick up an imaginary part for $s \geq 4 \mathrm{~m}^{2}$ and we claim that the global fit of S-, P- and D-wave phase shifts will allow one to fix the new free parameters 15$]$.

\section{APPENDIX:}

The polynomials multiplying the functions $g(s), G(s)$ and $Z(s)$ in Eq. (6) of second order correct by UPCA amplitudes are:

$$
\begin{aligned}
\pi^{2} p_{0}(s)= & \left(\frac{11}{72} \bar{\ell}_{1}+\frac{7}{36} \bar{\ell}_{2}+\frac{17}{48}\right) s^{2}-\left(\frac{5}{9} \bar{\ell}_{1}+\frac{5}{9} \bar{\ell}_{2}-\frac{1}{2} \bar{\ell}_{4}-\frac{95}{72}\right) s+ \\
& \frac{11}{18} \bar{\ell}_{1}+\frac{7}{9} \bar{\ell}_{2}-\frac{45}{8} \bar{\ell}_{3}-\frac{9}{2} \bar{\ell}_{4}+\frac{373}{144}, \\
\pi^{2} p_{1}(s)= & \frac{1}{432} \frac{1}{s-4}\left\{\left(6 \bar{\ell}_{1}-6 \bar{\ell}_{2}-2\right) s^{3}-\left(48 \bar{\ell}_{1}-48 \bar{\ell}_{2}-61\right) s^{2}+\right. \\
& \left.\left(96 \bar{\ell}_{1}-96 \bar{\ell}_{2}-197\right) s+120\right\}, \\
\pi^{2} p_{2}(s)= & \left(\frac{1}{36} \bar{\ell}_{1}+\frac{1}{9} \bar{\ell}_{2}+\frac{17}{96}\right) s^{2}-\left(\frac{1}{18} \bar{\ell}_{1}+\frac{7}{18} \bar{\ell}_{2}+\frac{1}{4} \bar{\ell}_{4}+\frac{61}{144}\right) s+ \\
& \frac{1}{9} \bar{\ell}_{1}+\frac{4}{9} \bar{\ell}_{2}-\frac{1}{8} \bar{\ell}_{3}+\frac{1}{2} \bar{\ell}_{4}+\frac{5}{18}, \\
q_{0}(s)= & -\frac{2}{9(s-4)}\left(50 s^{3}-260 s^{2}+303 s-36\right), \quad q_{1}(s)=-\frac{8}{9(s-4)^{2}}\left(6 s^{2}-55 s+64\right), \\
q_{2}(s)= & -\frac{4}{9(s-4)}\left(10 s^{3}-52 s^{2}+93 s-72\right), \quad r_{0}(s)=\frac{4}{3(s-4)}(6 s-25), \\
r_{1}(s)= & \frac{4}{3(s-4)^{2}}\left(3 s^{2}-13 s-6\right), \quad r_{2}(s)=-\frac{4}{3(s-4)}(3 s+1) .
\end{aligned}
$$

The polynomial part of QU2, $P_{I}$, in Eq. (6) are written as:

$$
\begin{aligned}
P_{I}(s) & =A_{I} s^{2}+B_{I} s+C_{I}, \quad \text { for } I=0 \text { and } 2 \\
(s-4) P_{1} & =A_{1} s^{3}+B_{1} s^{2}+C_{1} s+D_{1}
\end{aligned}
$$

with:

$$
\begin{aligned}
& \pi^{4} A_{0}=\frac{19}{2304} \bar{\ell}_{1}+\frac{13}{1152} \bar{\ell}_{2}+\frac{15}{1024} \bar{\ell}_{3}+\frac{11}{768} \bar{\ell}_{4}-\frac{21}{4096}+\frac{25 \pi^{2}}{576}-\frac{3083 \pi^{4}}{34560}+\frac{\pi^{4}}{2} \Phi_{0}^{\prime \prime}(4), \\
& \pi^{4} A_{1}=-\frac{1}{1152} \bar{\ell}_{1}+\frac{1}{1152} \bar{\ell}_{2}-\frac{13}{55296}-\frac{\pi^{2}}{452}-\frac{13 \pi^{4}}{30240}+\frac{\pi^{4}}{2} \Phi_{1}^{\prime \prime}(4), \\
& \pi^{4} A_{2}=\frac{1}{576} \bar{\ell}_{1}+\frac{7}{1152} \bar{\ell}_{2}+\frac{1}{3072} \bar{\ell}_{3}-\frac{1}{384} \bar{\ell}_{4}+\frac{\pi^{2}}{576}-\frac{131 \pi^{4}}{8640}+\frac{\pi^{4}}{2} \Phi_{2}^{\prime \prime}(4), \\
& \pi^{4} B_{0}=-\frac{61}{1152} \bar{\ell}_{1}-\frac{37}{576} \bar{\ell}_{2}-\frac{105}{512} \bar{\ell}_{3}-\frac{59}{384} \bar{\ell}_{4}-\frac{17 \pi^{2}}{48}+\frac{4331 \pi^{4}}{8640}+\pi^{4} \Phi_{0}^{\prime}(4)-4 \pi^{4} \Phi_{0}^{\prime \prime}(4), \\
& \pi^{4} B_{1}=\frac{1}{96} \bar{\ell}_{1}-\frac{1}{96} \bar{\ell}_{2}-\frac{29}{4608}+\frac{35 \pi^{2}}{288}+\frac{331 \pi^{4}}{30240}+\pi^{4} \Phi_{1}^{\prime \prime}(4)-6 \pi^{4} \Phi_{1}^{\prime \prime \prime}(4), \\
& \pi^{4} B_{2}=-\frac{5}{576} \bar{\ell}_{1}-\frac{11}{288} \bar{\ell}_{2}-\frac{7}{1536} \bar{\ell}_{3}+\frac{5}{384} \bar{\ell}_{4}-\frac{5 \pi^{2}}{48}+\frac{409 \pi^{4}}{4320}-\pi^{4} \Phi_{2}^{\prime}(4)-4 \pi^{4} \Phi_{2}^{\prime \prime}(4), \\
& \pi^{4} C_{0}=\frac{23}{288} \bar{\ell}_{1}+\frac{11}{144} \bar{\ell}_{2}+\frac{45}{128} \bar{\ell}_{3}+\frac{37}{96} \bar{\ell}_{4}+\frac{13 \pi^{2}}{18}-\frac{421 \pi^{4}}{720}+\pi^{4} \Phi_{0}(4)-4 \pi^{4} \Phi_{0}^{\prime}(4)+8 \pi^{4} \Phi_{0}^{\prime \prime}(4),
\end{aligned}
$$




$$
\begin{aligned}
& \pi^{4} C_{1}=-\frac{1}{24} \bar{\ell}_{1}+\frac{1}{24} \bar{\ell}_{2}-\frac{67}{1152}-\frac{67 \pi^{2}}{72}+\frac{53 \pi^{4}}{2520}+\pi^{4} \Phi_{1}^{\prime}(4)-8 \pi^{2} \Phi_{1}^{\prime \prime}(4)+24 \pi^{4} \Phi_{1}^{\prime \prime \prime}(4), \\
& \pi^{4} C_{2}=\frac{1}{144} \bar{\ell}_{1}+\frac{1}{18} \bar{\ell}_{2}+\frac{5}{384} \bar{\ell}_{3}-\frac{1}{96} \bar{\ell}_{4}+\frac{7 \pi^{2}}{18}-\frac{163 \pi^{4}}{720}+\pi^{4} \Phi_{2}(4)-4 \Phi_{2}^{\prime}(4)+8 \pi^{4} \Phi_{2}^{\prime \prime}(4), \\
& \pi^{4} D_{1}=\frac{1}{18} \bar{\ell}_{1}-\frac{1}{18} \bar{\ell}_{2}-\frac{157}{1728}+\frac{52 \pi^{2}}{27}-\frac{253 \pi^{4}}{840} .
\end{aligned}
$$

In order to compare the two approaches we have not included the dependence on $1 / F_{\pi}^{8}$ in the parameters $b_{i}$. In this way the quantities $b_{i}$ that we have used in Eqs. (7) stand for:

$$
\begin{aligned}
& b_{1}=8 \bar{\ell}_{1}+2 \bar{\ell}_{3}-2 \bar{\ell}_{4}+\frac{1}{48 \pi^{2}}\left(7 \ln \frac{m_{\pi}}{\mu}+\frac{13}{6}\right), \\
& b_{2}=-8 \bar{\ell}_{1}+2 \bar{\ell}_{4}-\frac{1}{12 \pi^{2}}\left(\ln \frac{m_{\pi}}{\mu}+\frac{1}{6}\right), \\
& b_{3}=2 \bar{\ell}_{1}+\frac{1}{2} \bar{\ell}_{2}-\frac{1}{16 \pi^{2}}\left(\ln \frac{m_{\pi}}{\mu}+\frac{7}{12}\right), \\
& b_{4}=\frac{1}{2} \bar{\ell}_{2}-\frac{1}{48 \pi^{2}}\left(\ln \frac{m_{\pi}}{\mu}+\frac{5}{12}\right), \\
& b_{5}=\frac{1}{16 \pi^{2}}\left[-\frac{31}{6} \bar{\ell}_{1}-\frac{145}{36} \bar{\ell}_{2}+\frac{7}{864}+\frac{1}{16 \pi^{2}}\left(\frac{625}{144} \ln \frac{m_{\pi}}{\mu}-\frac{66029}{20736}\right)\right]-\frac{21}{16} k_{1}-\frac{107}{96} k_{2}+r_{5}^{r}, \\
& b_{6}=\frac{1}{16 \pi^{2}}\left[-\frac{7}{18} \bar{\ell}_{1}-\frac{35}{36} \bar{\ell}_{2}+\frac{1}{432}+\frac{1}{16 \pi^{2}}\left(\frac{257}{432} \ln \frac{m_{\pi}}{\mu}-\frac{11375}{20736}\right)\right]-\frac{5}{48} k_{1}-\frac{25}{96} k_{2}+r_{6}^{r},
\end{aligned}
$$

noindent where:

$$
k_{1}=\frac{1}{192 \pi^{4}} \ln \frac{m_{\pi}}{\mu}\left(\bar{\ell}_{1}+\ln \frac{m_{\pi}}{\mu}\right) \quad k_{2}=\frac{1}{96 \pi^{4}} \ln \frac{m_{\pi}}{\mu}\left(\bar{\ell}_{2}+\ln \frac{m_{\pi}}{\mu}\right)
$$

and $\mu$ is the renormalization mass scale.

[1] I.S. Gerstein, J.H. Schnitzer and S. Weinberg, Phys. Rev. 170 (1968) 1638.

[2] S. Weinberg, Physica A 96 (1979) 327.

[3] J. Gasser and H. Leutwyler, Ann. Phys. 158 (1984) 142; Nucl. Phys. B250 (1985) 465;

[4] A. Dobado, M. J. Herrero and T. N. Truong, Phys. Lett. B 235 (1990) 134.

[5] A. Dobado and J.R. Peláez, Phys. Rev. D 47 (1993) 4883.

[6] G. Ecker, J. Gasser, A. Pich and E. De Rafael, Nucl. Phys. B 321 (1989) 311.

[7] J. Sá Borges, Nucl. Phys. B 51 (1973) 189.

[8] S. Weinberg, Phys. Rev. Lett. 17 (1966) 616.

[9] J. Sá Borges, Phys. Lett. B149 (1984) 21.

[10] J. Bijnens, G. Colangelo, G. Ecker, J. Gasser and M. E. Sainio, Phys. Lett. B374 (1996) 210 Pion-pion scattering at low energy, hep-ph/9707291.

[11] J. Sá Borges, J. Soares Barbosa and V. Oguri, Phys. Lett. B 393 (1997) 413.

[12] M. Knecht, B. Moussallam, J. Stern, and N. H. Fuchs, Nucl. Phys. 457 (1995) 513.

[13] J. Sá Borges and F.R.A. Simão, Phys. Rev. D 53 (1996) 4806.

[14] A.M. Bernstein and B.R. Holtein (eds.), Chiral Dynamics: Theory and Experiment. Proceedings of the Workshop held at MIT. Cambridge, MA. USA. 25/29 July 1994 (Springer, Berlin, Heidelberg, 1995);

B. Adeva et al.; Proposal to SPSLC: Lifetime measurement of $\pi^{+} \pi^{-}$atoms to test low energy QCD predictions, CERN/SPSLC/P 284, December 15 (1994).

[15] J. Sá Borges, J. Soares Barbosa and M. Tonasse, work in progress. 